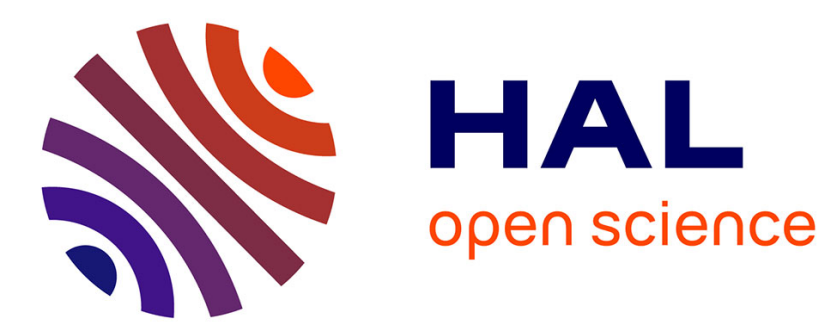

\title{
Unsupervised Classification and Characterization of Honeypot Attacks
}

Philippe Owezarski

\section{To cite this version:}

Philippe Owezarski. Unsupervised Classification and Characterization of Honeypot Attacks. 10th International Conference on Network and Service Management, Nov 2014, Rio de Janeiro, Brazil. hal-01070141

\section{HAL Id: hal-01070141 https://hal.science/hal-01070141}

Submitted on 30 Sep 2014

HAL is a multi-disciplinary open access archive for the deposit and dissemination of scientific research documents, whether they are published or not. The documents may come from teaching and research institutions in France or abroad, or from public or private research centers.
L'archive ouverte pluridisciplinaire HAL, est destinée au dépôt et à la diffusion de documents scientifiques de niveau recherche, publiés ou non, émanant des établissements d'enseignement et de recherche français ou étrangers, des laboratoires publics ou privés. 


\title{
Unsupervised Classification and Characterization of Honeypot Attacks
}

\author{
Philippe Owezarski*† \\ *CNRS, LAAS, 7 avenue du colonel Roche, F-31400 Toulouse, France \\ †Université de Toulouse, LAAS, F-31400 Toulouse, France
}

\begin{abstract}
Monitoring communication networks and their traffic is of essential importance for estimating the risk in the Internet, and therefore designing suited protection systems for computer networks. Network and traffic analysis can be done thanks to measurement devices or honeypots. However, analyzing the huge amount of gathered data, and characterizing the anomalies and attacks contained in these traces remain complex and time consuming tasks, done by network and security experts using poorly automatized tools, and are consequently slow and costly. In this paper, we present an unsupervised method for classification and characterization of security related anomalies and attacks occurring in honeypots. This as automatized as possible method does not need any attack signature database, learning phase, or labeled traffic. This corresponds to a major step towards autonomous security systems. This paper also shows how it is possible from anomalies characterization results to infer filtering rules that could serve for automatically configuring network routers, switches or firewalls.
\end{abstract}

Keywords-Honeypot attack detection, Anomaly classification, unsupervised machine learning, autonomous security systems.

\section{INTRODUCTION}

Monitoring communication networks and their traffic is an important advance for protecting computers systems. Several methods exist. For example, using monitoring devices at the interconnection points of networks allows the analysis of all incoming and outgoing flows. Another method consists in installing honeypots in the network. Honeypots provide more or less emulated services (according to the expected interaction level with the attackers), and allows supervising the use of the provided services by attackers. Thus, gathered data allows security experts on one hand to detect and analyze computer systems weaknesses, and on the other hand to collect useful information on the attacker activities that allows the analysis of their attacking methods, objectives, and strategies. Estimating and analyzing the risk related to illegitimate activities on the Internet are essential for security experts to design and develop adapted and efficient defense and protection systems with regard to the actual risk.

Characterizing and classifying current anomalies and attacks of the Internet are very complex and time consuming tasks, done by experts. Such tasks are therefore slow and costly. The main difficulty related to the identification and analysis of the different classes of illegitimate traffic is their fast evolution, amplification, and renewing capabilities. Designing autonomous classification and characterization processes is crucial for easy to deploy and to use defense systems. Hence, modern classification systems must not rely on human expert knowledge, and must be able to autonomously adapt to the evolution of all traffic components, be they legitimate or not (this paper obviously focuses on illegitimate ones). For this purpose, we propose to take advantage of unsupervised machine learning techniques that do not require the help of any human expert. In this paper we then present an unsupervised method for classifying and characterizing anomalies and attacks contained in traffic gathered on honeypot networks. This method does not take advantage of any attack signature, learning stage, or labeled traffic, what constitutes a major advance towards autonomous security systems. This approach uses robust clustering techniques, as sub-space clustering, density-based clustering, and evidence accumulation for classifying flow ensembles in traffic classes, and builds easily understandable associated signatures. This method was first proposed in our previous work [1] that aims at detecting, classifying and characterizing anomalies in the full network traffic. This method has been adapted and extended in order to cope with the specific honeypot network traffic. Indeed, honeypot network traffic only contains illegitimate traffic (attacks, anomalies, intrusion attempts, ...) what significantly changes the way classification and characterization can be done. This paper mainly deals with taking into account the illegitimate nature of traffic to be analyzed, and designing adapted and optimized classification and characterization algorithms.

These algorithms are illustrated by the analysis of honeypot traffic gathered at the University of Maryland, at the Netflow format [2]. The University of Maryland put measurement devices on routers at the border of the honeypots networks. This allows the capture of the traffic exchanged between honeypots and the Internet by monitoring only very few network devices. We nevertheless do not have the communication traces between the honeypots of the same network. The traffic traces we have been working on have been captured on a duration of more than one year.

The rest of the paper is as follows: section II presents related work in the domain of attack and anomalies detection and characterization, covering the full set of approaches from signature to statistical profile based, and finishing with approaches based on data mining and machine learning, especially unsupervised ones. This section also presents supervised and semi-supervised classification methods. Section III presents our previous unsupervised anomaly detection algorithm. This algorithm has already been published in [1]. We nevertheless add a presentation of this algorithm in this paper to make it self-contained. Interested readers can find an extended version of the algorithms description in [3]. However this algorithm originally works on full traffic, i.e. normal traffic on Internet links, and the objective is then to detect anomalies and attacks. 
Given the nature of the traffic we are considering in this paper - i.e. traffic between honeypots and the Internet - we know that it is completely illegitimate. This is a strong hypothesis that can help going further for characterizing and classifying the different component of this illicit traffic, and estimating the risk in the Internet. This section III then presents the sub-space clustering approach that aims at increasing the robustness of clustering algorithms, limiting its sensitivity, etc. It also presents the recombination mechanisms based on evidence accumulation and inter-clustering associations. As a strong improvement of the previous algorithm, section IV describes how we can show correlation existing between clusters in different subspaces, these correlations exhibiting that such clusters could correspond to the same attack or anomaly. This is a strong help for more accurately and completely identifying and characterizing all attacks contained in the honeypots traffic. Section $\mathrm{V}$ then proposes a way to automatically characterize the identified anomalies and attacks. It relies on the clustering and correlation results. Section VI proposes a method for ranking the risk an attack represents. Such ranking is aimed at helping security experts or network operators to prioritize their work, focusing first on the most dangerous ones. Section VII illustrates the results of this characterization algorithm by showing a set of attacks and anomalies that have been identified in the real traffic traces of the University of Maryland, and the characterizing signatures that have been issued. Last, section VIII concludes this paper.

\section{RELATED WORK}

The problem of network anomaly detection has been extensively studied during the last decade. Most of the approaches analyze statistical variations of traffic volume (e.g. number of packets, bytes or new flows) and/or traffic features (e.g. IP addresses and ports), using either single-link measurements or network-wide data. A non-exhaustive list of standard methods includes the use of signal processing techniques (e.g. ARIMA - Autoregressive Integrated Moving Average - modeling, wavelets-based filtering) on single-link traffic measurements [4], [5], PCA (Principal Component Analysis) for networkwide anomaly detection [6]-[8], and Sketches applied to IPflows [9], [10].

The simultaneous detection and characterization of traffic anomalies has also received quite a lot of attention in the past, but results are few and present important limitations, either because they rely on some kind of training data and/or anomaly signatures, or because they do not provide meaningful and tractable information to a human network operator, who has to take the final decision about the nature of the detected problem. Authors in [6] characterize network-wide anomalies in highly aggregated traffic (Origin-Destination flows or OD flows for short), using PCA and the sub-space approach [7]. An important limitation of this approach is that the information obtained from OD flow data is too coarse-grained to provide meaningful information to the network operator. Papers like Lakhina et al. [8] and Biang et al. [10] detect and characterize anomalies using finer-grained traffic information, basically applying the same PCA approach to the sample entropy of the empirical distribution of specific traffic features. One clear limitation of these approaches is that the information they provide is not immediately usable and easy-to-understand by the network operator, who may not even be familiar with concepts distant from his tasks such as sample entropy. Besides, the PCA approach is highly sensitive to noise when used for anomaly detection [11], [12], requiring in practice a fine-tuning and data-dependent calibration step to work.

UNADA (Unsupervised Network Anomaly detection Algorithm) [13] falls within the unsupervised anomaly detection domain, a novel research area that has drawn quite a lot of interest in the research community, but that still represents a rather immature field. Most work on unsupervised network anomaly detection has been devoted to the IDS field, generally targeting the detection of network intrusions in the very well known KDD'99 dataset. The great majority of the detection schemes proposed in the literature are based on clustering techniques and outliers detection, being [14]-[16] some examples. The objective of clustering is to partition a set of unlabeled patterns into homogeneous groups of "similar" characteristics, based on some similarity measure. Outliers detection consists in identifying those patterns that do not belong to any of these clusters. In [14], authors use a simple single-linkage hierarchical clustering method to cluster data from the KDD'99 dataset, based on the standard Euclidean distance for interpattern similarity. Eskin et al. [15] reports improved results in the same dataset, using three different clustering algorithms: the Fixed-Width clustering algorithm, an optimized version of the $k$-NN algorithm, and the one class SVM algorithm. Leung and Leckie [16] present a combined density-based and grid-based clustering algorithm to improve computational complexity, obtaining similar detection results.

Previous work of our own permits to automatically characterize network traffic anomalies [17], but using a-priori welldefined anomaly signatures. Closer to our current work, authors in [18] present URCA (Unsupervised Root Cause Analysis), a two-steps algorithm to characterize network anomalies in an unsupervised fashion. URCA uses as input the traffic in the anomalous time slots detected by any generic time-slotbased detection algorithm [19]. In the first step, it identifies the anomaly by iteratively removing from the anomalous time slots those flows that seem normal. In the second step, the algorithm uses a hierarchical clustering method to characterize the particular flows identified as anomalous. We identify some serious drawbacks and omissions in URCA: authors claim that the approach is unsupervised, which is not true, simply because it uses previously labeled anomalous events for the characterization. As in previous works, the algorithm uses difficult-to-interpret traffic descriptors for the clustering step (e.g. sample entropy of the distribution of IP addresses, aggregated at different levels), obscuring the comprehension of the network operator. Finally, the algorithm removes those flows that seem normal before the characterization step, which drags possible errors to the clustering step.

Our Unsupervised Anomaly Detection and Characterization algorithm [1] presents several advantages w.r.t. current state of the art. First and most important, it works in a completely unsupervised fashion, which means that it can be directly plugged into any monitoring system and start to work from scratch. Secondly, we perform anomaly detection based not only on outliers detection, but also by identifying small-clusters. This is achieved by using different levels of traffic aggregation, both at the source and destination of the traffic; this additionally permits to discover low-intensity 
and distributed anomalies. Thirdly, we avoid the lack of robustness of general clustering approaches, by combining the notions of Sub-Space Clustering [20] and multiple Evidence Accumulation [21]. In particular, our algorithm is immune to general clustering problems like sensitivity to initialization, specification of number of clusters, or structure-masking by irrelevant features. Fourthly, the algorithm performs clustering in low-dimensional feature spaces, using simple traffic descriptors like number of source IP addresses or fraction of SYN packets. This simplifies the characterization of the anomaly, and avoids well-known clustering problems when working with high-dimensional data [22]. Our algorithm ranks the multiple evidence of an anomaly detected in different subspaces, combining the most relevant traffic descriptors into a compact and easy-to-interpret signature that characterizes the problem. This permits to reduce the time spent by the network operator to understand the nature of the anomaly. Finally, this algorithm is designed to work in an on-line fashion, analyzing traffic from consecutive time slots in near real time. This is possible even when working with large number of traffic descriptors, because the sub-space clustering and the evidence accumulation algorithms are perfectly adapted for parallelization (see [1]).

To the best of our knowledge, there is no paper in the literature on the use of unsupervised classification and characterization algorithms on honeypot traffic, or for intrusion or attacks characterization on big data sets of attack traces. Some very recent work exists, especially by Symantec and the TRIAGE project (Data Analytics Framework for Intelligence Analysis) that aims to use autonomous data mining techniques for the analysis of all the gathered traces of attacks. However, TRIAGE aims at designing visualization techniques for the experts to make decision, while in this paper we propose to autonomously apply countermeasures for cheaper and faster defense.

\section{UNSUPERVISED ANOMALY DETECTION}

Our anomaly detection works on single-link packet-level traffic captured in consecutive time-slots of fixed length $\Delta_{T}$. The first analysis stage consists in change detection. At each time-slot, traffic is aggregated in 9 different flow levels $l_{i}$. These include (from finer to coarser-grained resolution): source IPs ( $l_{1}$ : IPsrc), destination IPs ( $l_{2}$ : IPdst), source Network Prefixes $\left(l_{3,4,5}\right.$ : IPsrc/24, /16, /8), destination Network Prefixes $\left(l_{6,7,8}\right.$ : IPdst $\left./ 24, / 16, / 8\right)$, and traffic per Time Slot ( $l_{9}$ : tpTS). Time series $Z_{t}^{l_{i}}$ are built for basic traffic metrics such as number of bytes, packets, and IP flows per time slot, using the 9 flow resolutions $l_{1 \ldots 9}$. Analyzing honeypot traffic at multiple aggregation levels permits to detect both single source-destination and distributed attacks of very different intensities.

The unsupervised anomaly detection stage takes as input all the flows in the time slot flagged as anomalous, aggregated according to one of the different levels used in the first stage. An anomaly will generally be detected in different aggregation levels, and there are many ways to select a particular aggregation to use in the unsupervised stage; for the sake of simplicity, we shall skip this issue, and use any of the aggregation levels in which the anomaly was detected. Without loss of generality, let $\mathbf{Y}=\left\{\mathbf{y}_{1}, . ., \mathbf{y}_{F}\right\}$ be the set of $F$ flows in the flagged time slot, referred to as patterns in more general terms. Each flow $\mathbf{y}_{f} \in \mathbf{Y}$ is described by a set of $A$ traffic attributes or features. In this paper, we use a list of common traffic attributes. The list includes $A=9$ traffic features: number of source/destination IP addresses and ports, ratio of number of sources to number of destinations, packet rate, ratio of packets to number of destinations, and fraction of ICMP and SYN packets. According to our previous work on signature-based anomaly characterization [17], such simple traffic descriptors permit characterization of general traffic anomalies in easy-tointerpret terms. The list is therefore by no means exhaustive, and more features can be easily plugged-in to improve results. Let $\mathbf{x}_{f}=\left(x_{f}(1), . ., x_{f}(A)\right) \in \mathbb{R}^{A}$ be the corresponding vector of traffic features describing flow $\mathbf{y}_{f}$, and $\mathbf{X}=\left(\mathbf{x}_{1} ; . . ; \mathbf{x}_{F}\right)$ the complete matrix of features, referred to as the feature space.

The unsupervised detection algorithm is based on clustering techniques applied to $\mathbf{X}$. The objective of clustering is to partition a set of unlabelled patterns into homogeneous groups of similar characteristics, based on some measure of similarity. Table I explains the characteristics of each anomaly in terms of type, distributed nature, aggregation type and netmask used, and impact on traffic features. On one hand, a SYN DDoS which targets one machine from a high number of hosts located in several /24 addresses will constitute a cluster if flows are aggregated in $l_{3}$. In fact, each of these $/ 24$ addresses will have traffic attributes values different from the ones of normal traffic: a high number of packet, a single destination and many SYN packets. It is the whole set of these flows that will create a cluster. On the other hand, if flows are aggregated in $l_{6}$, the only destination address will be an outlier characterized by many sources and a high proportion of SYN packets.

Our particular goal is to identify and to isolate the different flows that compose the anomaly flagged in the first stage, both in a robust way. Unfortunately, even if hundreds of clustering algorithms exist [22], it is very difficult to find a single one that can handle all types of cluster shapes and sizes, or even decide which algorithm would be the best for our particular problem. Different clustering algorithms produce different partitions of data, and even the same clustering algorithm provides different results when using different initializations and/or different algorithm parameters. This is in fact one of the major drawbacks in current cluster analysis techniques: the lack of robustness.

To avoid such a limitation, we have developed a divide and conquer clustering approach, using the notions of clustering ensemble [23] and multiple clusterings combination. A clustering ensemble $\mathbf{P}$ consists of a set of $N$ partitions $P_{n}$ produced for the same data with $n=1, \ldots, N$. Each of these partitions provides a different and independent evidence of data structure, which can be combined to construct a global clustering result for the whole feature space. There are different ways to produce a clustering ensemble. We use Sub-Space Clustering (SSC) [20] to produce multiple data partitions, applying the same clustering algorithm to $N$ different subspaces $\mathbf{U}_{n} \subset \mathbf{X}$ of the original space.

\section{A. Clustering Ensemble and Sub-Space Clustering}

Each of the $N$ sub-spaces $\mathbf{U}_{n} \subset \mathbf{X}$ is obtained by selecting $R$ features from the complete set of $A$ attributes. The number of sub-spaces $N$ hence is equal to $R$-combinations-obtainedfrom- $A$. To set the sub-space dimension $R$, we take a very 
TABLE I. FEATURE USED FOR THE DETECTION OF DOS, DDOS, NETWORK/PORT SCANS, AND SPREADING WORMS. ANOMALIES OF DiSTRIBUTED NATURE 1-TO-N OR N-TO-1 INVOLVE SEVERAL /24 (SOURCE OR DESTINATIONS) ADDRESSES CONTAINED IN A SINGLE /16 ADDRESS.

\begin{tabular}{|c|c|c|c|c|}
\hline Anomaly & Distributed nature & Aggregation type & Clustering result & Impact on traffic features \\
\hline \multirow{2}{*}{ DoS (ICMP $\vee S Y N)$} & \multirow[t]{2}{*}{ 1-to-1 } & IPsrc/* & Outlier & \multirow{2}{*}{$\begin{array}{l}\text { nSrcs }=\text { nDsts }=1, \text { nPkts } / \text { sec }>\lambda_{1}, \text { avgPktsSize }<\lambda_{2}, \\
\left(\mathrm{nICMP} / \mathrm{nPkts}>\lambda_{3} \vee \mathrm{nSYN} / \mathrm{nPkts}>\lambda_{4}\right)\end{array}$} \\
\hline & & IPdst/* & Outlier & \\
\hline \multirow{3}{*}{$\begin{array}{l}\mathrm{DDoS}(\mathrm{ICMP} \vee \mathrm{SYN}) \\
\text { to several @IP/24 }\end{array}$} & \multirow{3}{*}{ N-to-1 } & IPsrc/24 $\left(l_{3}\right)$ & Cluster & \multirow{3}{*}{$\begin{array}{l}\text { nDsts }=1, \text { nSrcs }>\alpha_{1}, \text { nPkts } / \text { sec }>\alpha_{2}, \text { avgPktsSize }<\alpha_{3}, \\
\left(\mathrm{nICMP} / \mathrm{nPkts}>\alpha_{4} \vee \mathrm{nSYN} / \mathrm{nPkts}>\alpha_{5}\right) .\end{array}$} \\
\hline & & IPsrc/16 $\left(l_{4}\right)$ & Outlier & \\
\hline & & IPdst/* & Outlier & \\
\hline \multirow{2}{*}{ Port scan } & \multirow{2}{*}{ 1-to-1 } & IPsrc/* & Outlier & \multirow{2}{*}{$\begin{array}{l}\mathrm{nSrcs}=\mathrm{nDsts}=1, \mathrm{nDstPorts}>\beta_{1}, \text { avgPktsSize }<\beta_{2}, \\
\mathrm{nSYN} / \mathrm{nPkts}>\beta_{3} .\end{array}$} \\
\hline & & IPdst/* & Outlier & \\
\hline \multirow{3}{*}{$\begin{array}{l}\text { Network scan to } \\
\text { several @IP/24 }\end{array}$} & \multirow{3}{*}{ 1-to-1 } & IPsrc/* & Outlier & \multirow{3}{*}{$\begin{array}{l}\mathrm{nSrcs}=1, \mathrm{nDsts}>\delta_{1}, \text { nDstPorts }>\delta_{2}, \text { avgPktsSize }<\delta_{3}, \\
\mathrm{nSYN} / \mathrm{nPkts}>\delta_{4}\end{array}$} \\
\hline & & IPdst/24 $\left(l_{6}\right)$ & Cluster & \\
\hline & & IPdst/16 $\left(l_{7}\right)$ & Outlier & \\
\hline \multirow{3}{*}{$\begin{array}{l}\text { Spreading worms to } \\
\text { several @IP/24 }\end{array}$} & \multirow{3}{*}{$1-$ to-N } & IPsrc/* & Outlier & \multirow{3}{*}{$\begin{array}{l}\mathrm{nSrcs}=1, \text { nDsts }>\eta_{1}, \text { nDstPorts }<\eta_{2}, \text { avgPktsSize }<\eta_{3}, \\
\mathrm{nSYN} / \text { nPkts }>\eta_{4}\end{array}$} \\
\hline & & IPdst/24 $\left(l_{6}\right)$ & Cluster & \\
\hline & & IPdst/16 $\left(l_{7}\right)$ & Outlier & \\
\hline
\end{tabular}

useful property of monotonicity in clustering sets, known as the downward closure property: "if a collection of points is a cluster in a $d$-dimensional space, then it is also part of a cluster in any $(d-1)$ projections of this space" [24]. This directly implies that, if there exists any evidence of density in $\mathbf{X}$, it will certainly be present in its lowest-dimensional sub-spaces. Using small values for $R$ provides several advantages: firstly, doing clustering in low-dimensional spaces is more efficient and faster than clustering in bigger dimensions. Secondly, density-based clustering algorithms provide better results in low-dimensional spaces [24], because high-dimensional spaces are usually sparse, making it difficult to distinguish between high and low density regions. We shall therefore use $R=2$ in our SSC algorithm, which gives $N=C_{R}^{A}=A(A-1) / 2$ partitions.

\section{B. Combining Multiple Partitions}

Having produced the $N$ partitions, we now explore different methods to combine these partitions in order to build a single partition where anomalous flows are easily distinguishable from normal-operation traffic: the classical Evidence Accumulation (EA) and the new Inter-Clustering Result Association (ICRA) method.

1) Combining Multiple Partitions using Evidence Accumulation: A possible answer is provided in [21], where authors introduced the idea of multiple-clusterings Evidence Accumulation (EA). By simple definition of what it is, an anomaly may consist of either outliers or small-size clusters, depending on the aggregation level of flows in $\mathbf{Y}$ ( $\mathrm{cf}$ table I). EA then uses the cluster ensemble $\mathbf{P}$ to build two inter-pattern similarity measures between the flows in $\mathbf{Y}$. These similarity measures are stored in two elements: a similarity matrix $S$ to detect small clusters and a vector $D$ used to rank outliers. $S(p, q)$ represents the similarity between flows $p$ and $q$. This value increases when the flows $p$ and $q$ are in the same cluster many times and when the size of this cluster is small. These two parameters allows the algorithm to target small clusters. $D(o)$ represents the abnormality of the outlier $o$. This value increases when the outlier has been classified as such several times and when the separation between the outlier and the normal traffic is important. As we are only interested in finding the smallestsize clusters and the most dissimilar outliers, the detection consists in finding the flows with the biggest similarity in $S$ and the biggest dissimilarity in $D$. Any clustering algorithm can then be applied on the matrix $S$ values to obtain a final partition of $\mathbf{X}$ that isolates small-size clusters of close similarity values. A variable detection threshold over the values in $S$ is also able to detect small-size cluster. Concerning dissimilar outliers, they can be isolated though a threshold applied on the values in $D$.

2) Combining Multiple Partitions using Inter-Clustering result Association: However, by reasoning over the similarities between patterns (here flows), EA introduces several potential errors. Let us consider two pattern sets $P_{i}$ and $P_{j}$, if the cardinality of these pattern sets is close and if they are present in a similar number of sub-spaces, then EA will produce a very close (potentially the same) similarity value for both flow sets. They will then likely be falsely considered as belonging to the same cluster. This possibility has to be considered very seriously as it can induce a huge error: different anomalies will be merged together and will then likely be wrongly identified and characterized. Another source of potential error when using a clustering algorithm over $S$ values is the algorithm sensitivity to wrong parameters. Furthermore, the use of a threshold over $S$ and/or $D$ can decrease the system performance in case of a wrong value used.

In order to avoid the previously exposed sources of error, we introduce a new way of combining clustering results obtained from sub-spaces: Inter-Clustering Results Association. The idea is to address the problem in terms of cluster of flows and outlier of flow similarity instead of pattern (or flow) similarity. Hence, we shift the similarity measure from the patterns to the clustering results. The problem can then be split in two sub-problems: correlate clusters through Inter-CLuster Association (ICLA), and correlate outlier through Inter-Outlier Association (IOA).

In each case, a graph is used to express similarity between either clusters or outliers. Each vertex is a cluster/outlier from any sub-space $\mathbf{U}_{n}$ and each edge represents the fact that two connected vertices are similar. The underlying idea is straightforward: identify clusters or outliers present in different sub-spaces that contain the same flows. To do so, we first define a cluster similarity measure called $C S$ between two clusters $C_{r}$ and $C_{s}: C S\left(C_{r}, C_{s}\right)=\frac{\operatorname{card}\left(C_{r} \cap C_{s}\right)}{\max \left(\operatorname{card}\left(C_{r}\right), \operatorname{card}\left(C_{s}\right)\right.}$, card being the function that associates a pattern set with its cardinality, and $C_{r} \cap C_{s}$ the intersection of $C_{r}$ and $C_{s}$. Each edge in the cluster similarity graph between two $C_{r}$ and $C_{s}$ means $C S\left(C_{r}, C_{s}\right)>0.9$, being this an empirically chosen value. The value 0.9 guarantees that the vast majority of patterns are located in both clusters with a small margin of error. IOA uses an outlier similarity graph built by linking every outlier to every other outlier that contains the same pattern. Once these graphs are built, we need to find cluster sets where every cluster contains the same flows. In terms of 
vertices, we need to find vertex sets where every vertex is linked to every other vertex. In graph theory, such vertex set is called a clique. The clique search problem is a NP-hard problem. Most existing solutions use exhaustive search inside the vertex set which is too slow for our application. We then make the hypothesis that a vertex can only be part of a single clique. A greedy algorithm is then used to build each clique. Anomalous flow set are finally identified as the intersection of all the flow sets present in the clusters or outliers within each clique.

\section{CORRELATING ANOMALOUS TRAFFIC CLASSES}

\section{A. Address related correlation}

Thanks to previous algorithm, we can detect several classes of illegitimate traffic, but these classes can appear in different aggregation levels. We know that such classes present at different levels can be related to each other. Indeed, two traffic classes in two different aggregation levels are related for example when their flows come from the same sources and go towards the same destinations. For a better characterization of traffic classes, it is then important to link classes corresponding to the same single anomaly. Correlating illegitimate traffic classes is a solution for that purpose; it is able to determine the similarity between classes at each aggregation level, for possibly grouping them if they belong to the same anomaly.

For estimating the similarity between two illegitimate traffic classes on two different aggregation levels, we use a comparison function. It relies on IP addresses comparison [1]. The comparison method then uses the IP source and destination addresses of the different traffic classes $a_{1}$ and $a_{2}$. It then compares source IP addresses with each other, and destination IP addresses with each other, using function (1).

$$
\operatorname{Sim}_{@}\left(@_{1}, @_{2}\right)=\frac{\left|@_{1} \cap @_{2}\right|}{\max \left(\left|@_{1}\right|,\left|@_{2}\right|\right)}
$$

where $@_{n}$ is a set of IP addresses, and $\left|@_{n}\right|$ the number of addresses in this set.

It exists a similarity between two illegitimate traffic classes if equation (2) is true. tTSAddrSims is a threshold to be defined.

$$
\begin{aligned}
& \operatorname{Sim}_{\text {src }}\left(@_{1}, @_{2}\right)>t T S A d d r \operatorname{Sims} \wedge \operatorname{Sim}_{\text {dest }}\left(@_{1}, @_{2}\right) \\
& >t T S A d d r S i m s
\end{aligned}
$$

This method as defined in our previous work [1] has some lacks, and especially because it does not consider time. It appears when testing this method on the Maryland data that some flows of the same class are separated in time by several months. It is then clear that it is not satisfactory to consider only IP addresses. For example, illegitimate traffic classes detailed in tables II and III are completely different from a behavior point of view. For the first class, the number of sent packets in each flow is around 54, whereas it is around 4 in the second class. In addition, the time difference between these sendings is around two months. The only similarity between these two classes is related to their source IP addresses (saddr), and destination IP addresses (daddr). In this case the similarity value between the two classes with the previous function is $66 \%$, what is significantly high, and would indicate a strong link between them. Given the time at which they happened it is certainly not true, and malware infecting the machines are certainly not the same. As a consequence, we added temporal features to the correlation function.

Let us consider two illegitimate traffic classes $a_{1}$ and $a_{2}$. $T_{1}$ and $T_{2}$ are the set of time intervals in which traces of $a_{1}$ and $a_{2}$ appear. $@_{1}^{n}$ and $@_{2}^{n}$ are the set of source and destination IP addresses of classes $a_{1}$ and $a_{2}$ for the interval $t_{n} \in T_{1}$. The new similarity function is then defined as:

$$
\text { SimTime@ }=\sum_{t \in T_{1}} \frac{\operatorname{Sim}_{@}\left(@_{1}^{t}, @_{2}^{t}\right)}{\max \left(\left|@_{1}^{t}\right|,\left|@_{2}^{t}\right|\right)}
$$

However, if it exists time intervals between two classes, the similarity function equals a value very close from zero, whereas these two classes can be highly similar for other features. We then still need to keep in the computing of the similarity function the IP addresses of the anomaly classes $a_{1}$ and $a_{2}$. But the addresses must be computed intependentely from any temporal feature.

$$
\operatorname{SimGlobal}_{@}=\frac{\operatorname{Sim}_{@}\left(@_{1}, @_{2}\right)}{\max \left(\left|@_{1}\right|,\left|@_{2}\right|\right)}
$$
(5)

We then obtain the simlarity function defined by equation

$$
\begin{aligned}
& \operatorname{SimAnomalies}(a 1, a 2)=\left(\text { SimTime }_{\operatorname{Src}}\left(a_{1}, a_{2}\right)>\lambda_{1}\right) \\
& \wedge\left(\operatorname{SimTime}_{\text {Dest }}\left(a_{1}, a_{2}\right)>\lambda_{1}\right) \\
& \wedge\left(\operatorname{SimGlobal}_{\operatorname{Src}}\left(a_{1}, a_{2}\right)>\lambda_{2}\right) \\
& \wedge\left(\operatorname{SimGlobal}_{\text {Dest }}\left(a_{1}, a_{2}\right)>\lambda_{2}\right)
\end{aligned}
$$

The result of this function is a boolean value which is true if $a_{1}$ and $a_{2}$ are similar.

\section{B. Time related correlation}

Let's take again the examples of classes detailed in tables II and III. It is clear that there is no link between these two classes because they happened at very different times. Let's apply equation (5). Let's consider IP addresses of the first class on February 1st at $2 \mathrm{~h} 40$ am. Source IP addresses are 192.168.0.1 and 192.168.0.2. At that time, the second class is empty (it happened on April 10th to 13th). The similarity is then correctly estimated as false. By continuing with other times and the same method, we always obtain a false value for the similarity. The new similarity function then correctly does not find any similarity between these two illegitimate traffic classes.

Nevertheless, it is required with this new method to fix correct threshold values. If thresholds are not well selected returned values could be erroneous. With a small $\lambda_{1}$ value, each time a small temporal similarity will appear between two illegitimate classes, it will be the global similarity value that will determine the final similarity result between the two classes. If $\lambda_{1}$ is high, the two classes will be considered as similar if they sent packet almost at the same time, from the same source and to the same destinations. This is a strong 


\begin{tabular}{|c|c|c|c|c|c|c|c|c|}
\hline month & day & hour & min & saddr & daddr & nPkts & nBytes & nSyn/nPkts \\
\hline 02 & 01 & 02 & 4 & $192.168 .0 .1,192.168 .0 .2$ & $172.16 .4 .16,172.16 .4 .21$ & 54 & 4726 & 0.1481481 \\
\hline 02 & 01 & 03 & 40 & $192.168 .0 .1,192.168 .0 .3$ & $172.16 .4 .16,172.16 .4 .21$ & 54 & 4869 & 0.1481481 \\
\hline 02 & 01 & 18 & 05 & $192.168 .0 .1,192.168 .0 .3$ & $172.16 .4 .16,172.16 .4 .21$ & 53 & 3996 & 0.0754717 \\
\hline 02 & 01 & 19 & 55 & $192.168 .0 .1,192.168 .0 .2$ & $172.16 .4 .16,172.16 .4 .21$ & 54 & 4545 & 0.1481481 \\
\hline
\end{tabular}

TABLE II. FIRST EXAMPLE OF AN ILLEGITIMATE TRAFFIC CLASS (IP ADDRESSES HAVE BEEN ANONYMISED)

\begin{tabular}{|c|c|c|c|c|c|c|c|c|}
\hline month & day & hour & min & saddr & daddr & nPkts & nBytes & nSyn/nPkts \\
\hline 04 & 10 & 01 & 30 & $192.168 .0 .1,192.168 .0 .2$ & $172.16 .4 .16,172.16 .4 .21$ & 5 & 20 & 1 \\
\hline 04 & 11 & 01 & 30 & $192.168 .0 .1,192.168 .0 .3$ & $172.16 .4 .16,172.16 .4 .21$ & 4 & 160 & 1 \\
\hline 04 & 12 & 01 & 30 & $192.168 .0 .1,192.168 .0 .2$ & $172.16 .4 .16,172.16 .4 .21$ & 5 & 20 & 1 \\
\hline 04 & 13 & 01 & 30 & 192.168 .0 .1 & $172.16 .4 .16,172.16 .4 .21$ & 4 & 160 & 1 \\
\hline
\end{tabular}

constraint and forbids any time difference in the sendings. The global similarity will then be of less importance. Based on our experience with the traces of University or Maryland, we empirically recommend to select for $\lambda_{1}$ a low threshold value, between $10 \%$ and $30 \%$, and for $\lambda_{2}$ a threshold value greater than $25 \%$.

Illegitimate traffic classes that appear as different after the sub-space clustering phase can then be grouped, as it is shown that they correspond to the same anomaly.

\section{Automatic Characterization of Anomalies}

At this stage, the global traffic from and towards honeypots has been decomposed into traffic classes that exhibit different behaviors. Classification techniques of the Internet traffic have now to identify the type of each of these traffic classes, the generating application, or the type of attack or anomaly, each of these clusters is related to. Most advanced classification techniques, i.e. semi-supervised techniques, take advantage of signatures that specifically identify one of the possible traffic families, applications, or attacks. These signatures come either from a previous knowledge or expertise in this domain, either on a training stage on a known traffic, whose application components have already been labeled. This is obviously a strong requirement for classification purposes, but it remains a severe limit for designing a fully autonomous method.

However, in this work, at the opposite of what has been already done in the research area of autonomous traffic classification, this constraint does not exist because of the traffic nature that we have to analyze: illegitimate traffic. It is thus not needed to perfectly identify the anomaly kind (or attack), or to name it. Indeed, all traffic classes that have been isolated by the sub-space clustering algorithm, and evidence accumulation are anomalies or attacks. Therefore, the computing to be performed for each traffic anomaly or attack, in fine, consists in discarding them, after having identified any necessary feature required for instance for estimating the risk they represent in the Internet.

We then propose for this purpose to automatically generate the rules characterizing the anomaly classes. Based on these rules, it is easy to understand the anomalies characteristics and to infer the countermeasures to be performed (and of course, this can be done by a computer process).

At this stage, the sub-space clustering / evidence accumulation / correlation algorithm has identified several correlated anomalies containing a set of traffic flows in $\mathbf{Y}$ far out the rest of the traffic. The following task is to produce the appropriate filtering rules to correctly isolate and characterize each of these anomalies.

In order to produce filtering rules, the algorithm selects those sub-spaces $\mathbf{U}_{n}$ where the separation between the considered anomalous flows and the rest of the traffic is the biggest. We define two different classes of filtering rule: absolute rules $F R_{A}(\mathbf{Y})$ and relative rules $F R_{R}(\mathbf{Y})$. Absolute rules do not depend on the separation between flows, and correspond to the presence of dominant features in the considered flows. An absolute rule for a certain feature $j$ characterizing a certain flow set $Y_{g}$ has the form

$$
F R_{A}\left(\mathbf{Y}_{g}, a\right)=\left\{\forall \mathbf{y}_{f} \in \mathbf{Y}_{g} \subset \mathbf{Y}: x_{f}(a)==\lambda\right\} .
$$

For example, in the case of an ICMP flooding attack, the vast majority of the associated flows use only ICMP packets, hence the absolute filtering rule $\{\mathrm{nICMP} / \mathrm{nPkts}==1\}$ makes sense. On the contrary, relative filtering rules depend on the relative separation between anomalous and normaloperation flows. Basically, if the anomalous flows are well separated from the normal cluster in a certain partition $P_{n}$, then the features of the corresponding sub-space $\mathbf{U}_{n}$ are good candidates to define a relative filtering rule. A relative rule has the form $\lambda\}$.

$$
F R_{R}\left(\mathbf{Y}_{g}, a\right)=\left\{\forall \mathbf{y}_{f} \in \mathbf{Y}_{g} \subset \mathbf{Y}: x_{f}(a)<\lambda \vee x_{f}(a)>\right.
$$

We shall also define a covering relation between filtering rules: we say that rule $f_{1}$ covers rule $f_{2} \Leftrightarrow f_{2}(\mathbf{Y}) \subset f_{1}(\mathbf{Y})$. If two or more rules overlap (i.e., they are associated to the same feature), the algorithm keeps the one that covers the rest.

In order to construct a compact signature of the anomaly, we have to devise a procedure to select the most discriminant filtering rules. Absolute rules are important, because they define inherent characteristics of the anomaly. As regards relative rules, their relevance is directly tied to the degree of separation between anomalous and normal flows. In the case of outliers, we select the $K$ features for which the Mahalanobis distance to the normal-operation traffic is among the top- $K$ biggest distances. In the case of small-size clusters, we rank the relatives rules according to the degree of separation to the normal anomaly using the well-known Fisher Score (FS) which uses the variance in each cluster (normal and anomalous). To finally construct the signature, the absolute rules and the top$K$ relative rules are combined into a single inclusive predicate, using the covering relation in case of overlapping rules. 


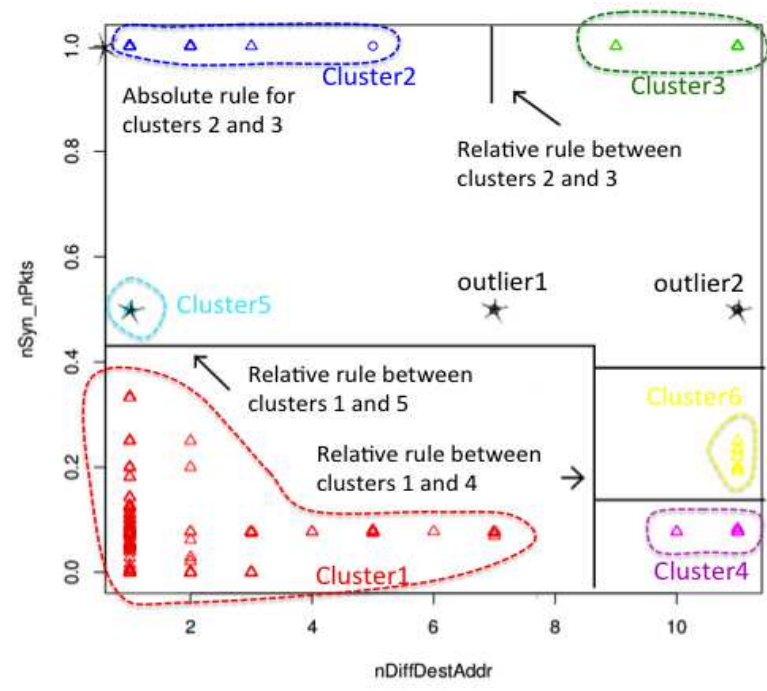

Fig. 1. The different filtering rules for sub-spaces (nSyn/nPkts, nDiffDestAddr)

What follows gives a real example of the generation of filtering rules based on an anomaly characterization. It is depicted on Figure 1. Clusters 2 and 3 have their value $\mathrm{nSyn} / \mathrm{nPkts}$ always equal to 1 . It then exists for them an absolute rule $\{n$ Syn $/ n$ Pkts $==1\}$.

On Figure 1, there exists a relative rule between cluster 1 and cluster 4 . For generating it, we draw the median between clusters 1 and 4 . Based on this median value, we can create the relative rule $\{n D$ iff $D$ est $A d d r<9\}$.

\section{RISK BASED ANOMALIES RANKING}

Estimating the risk related to an anomaly can help a security expert, in the general case, selecting the traffic classes that require to be computed in priority. In our specific case where all traffic classes identified are illegitimate, it can help to distinguish for instance between anomalies that are real attacks from scanning which in general just serves for preparing a possible future attack. In that case, the risk ranking can help superposing relative filtering rules, targeting in priority the most risky one.

The way our risk ranking is done depends on the amount of communication; the more communication in an anomaly or attack, the more risky. In addition, anomalies that appear in many sub-spaces are considered as more dangerous than the ones appearing in a single or on very few sub-spaces. Three features are considered for estimating the risk related to an anomaly. The first one is the number of packets of the anomaly, because, if there are many packets exchanged between the attacking and the victim machines, there exist potentially machines infected by a virus, or a flooding attack attempt. The second feature is the amount of bytes exchanged in the anomaly, because, even if the number of exchanged packets is reduced, a large amount of bytes could have been exchanged. This may correspond to the download of information from a victim machine by an attacker, or the upload of viruses on several victim machines from the attacker machine. Last, the third feature is the communication duration between the attacker and a target machine. The longer the communication duration, the more probable an attacker performing a download, or having an open shell on the victim machine.

The formula used for the risk estimation is:

risk $=C *(\log (n$ Pkts $)+\log ($ nBytes $)+\log ($ duration +1$))$

where $C$ is the number of sub-spaces in which the anomaly appears, nPkts is the number of exchanged packets in the anomaly, nBytes is the number of exchanged bytes, and duration is the duration of the anomaly. +1 appears in $\log ($ duration +1$)$ for avoiding some errors as duration $\in \mathbb{R}_{+}$ whereas (nPkts, nBytes) $\in \mathbb{R}_{+}^{*}$. The communication duration can be assimilated as zero because it can be so small that measurement devices can measure it as zero. On the other side, the number of packets or bytes are necessarily greater or equal to 1 .

\section{EXPERIMENTAL EVALUATION IN REAL TRAFFIC}

We run the algorithm described in this paper on the honeypot traffic traces gathered at the University of Maryland. For obvious privacy reasons, as well as space limit, we will not present the complete set of attacks evidenced. But we will show on an example how the algorithm behaves, and how it succeed in classifying attacks and anomalies, how it builds the anomaly classes characteristics based on sub-space clustering, evidence accumulation, and anomaly correlation. It also presents the filtering rules that have been autonomously generated and that can serve for automatically configuring security devices as filtering functions of routers, or firewalls.

This section starts by showing the complete behavior of the algorithm applied on a single anomaly (designated as anomaly [224]). Figure 2 shows 5 sub-spaces in which anomalies mentioned in this section appear (all sub-spaces in which the anomaly appears are not depicted on figure 2 for space limit reason). On each of these sub-spaces, clusters clearly appear, each corresponding to different anomalies. Figure ?? shows the cliques that have been built for correlating the clusters found in different sub-spaces, and then linking the ones that correspond to the same anomaly or attack.

Anomaly [224] appears in Figure 2 on subspaces (c) and (e), on the red cluster with $n$ Rst ${ }_{n}$ Pkts $=0$.

Finally, the algorithm generated the following signature that fully characterizes anomaly [224]:

\section{Characteristics of attack [224]:}

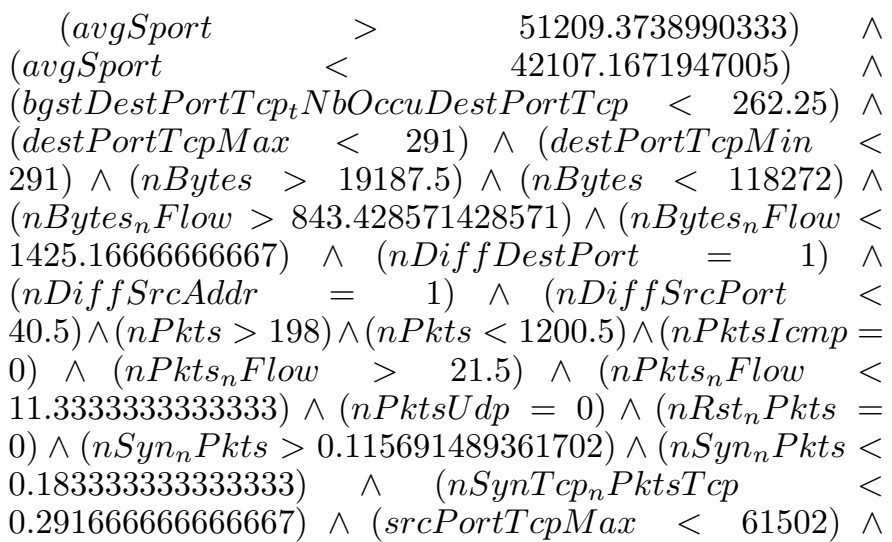




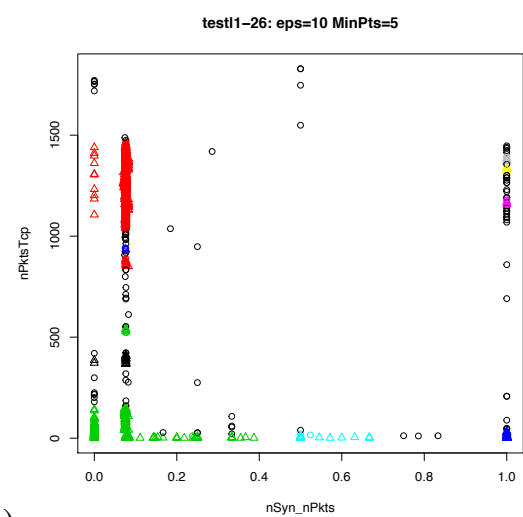

(a)

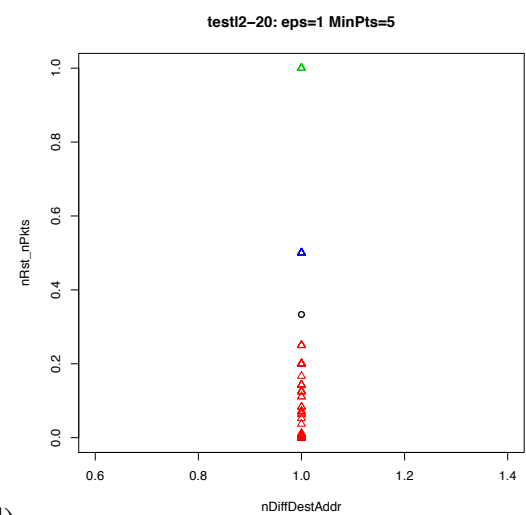

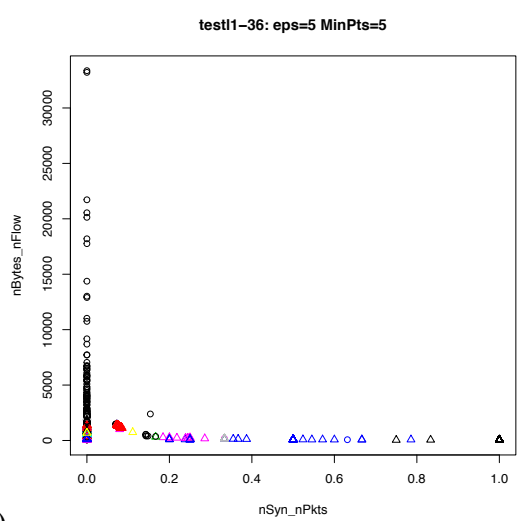

(b)

(c)
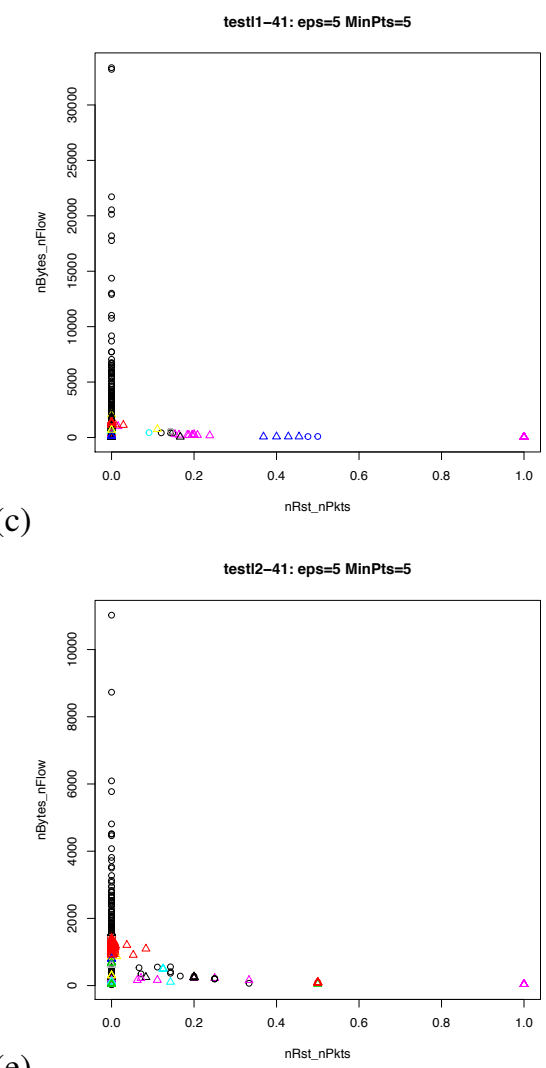

(e)

Fig. 2. Sub-spaces in which anomalies [44], [224], and [327] appear. These sub-spaces correspond to different IP address aggregation levels and different temporal granularities
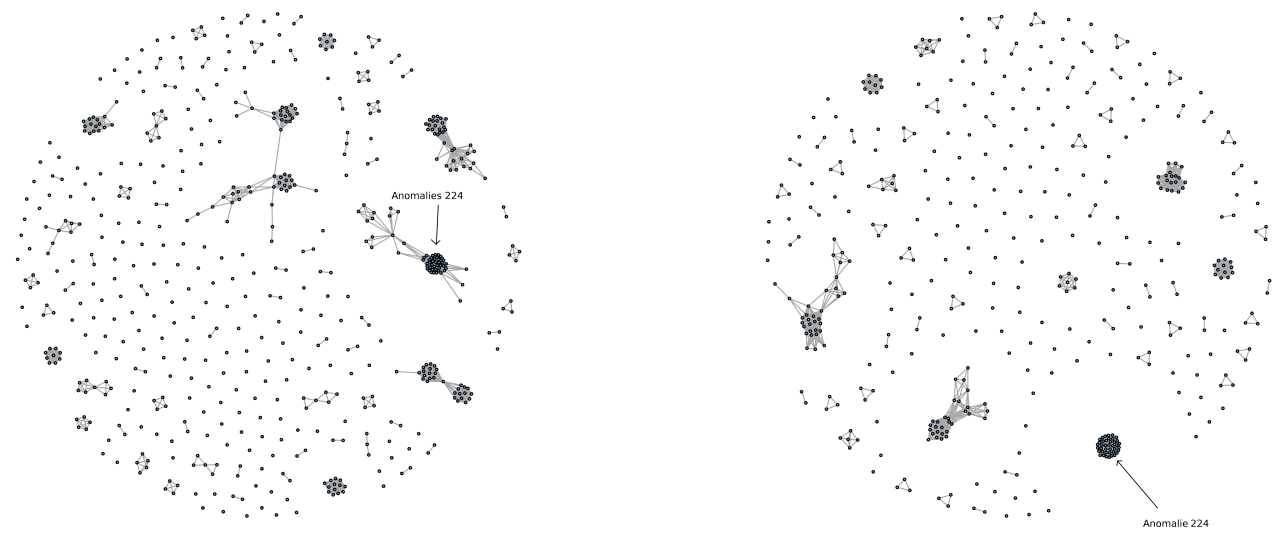

Fig. 3. Two levels of cliques appearing when correlating anomalies in different sub-spaces

$($ srcPortTcpMax > 34033.5) ^( srcPortTcpMin < $61502) \wedge(\operatorname{srcPortTcpMin}>34033.5)$

Anomaly [327] appears in Figure 2 on subspaces (d) with the blue cluster, and (e), with the green cluster corresponding to RRst $_{n}$ Pkts $=0.5$.
Finally, the algorithm generated the following signature that fully characterizes anomaly [327]:

\section{Characteristics of attack [327]:}

$\begin{gathered}\left(\begin{array}{l}\text { nBytes } \\ n\end{array} \text { Flow }\right. \\ \left(\text { nBytes }{ }_{n} \text { Flow }\right.\end{gathered}>\quad \begin{gathered}93.6666666666667) \\ (\text { nDiffSrcAddr }\end{gathered} \wedge$


1) $\wedge\left(\right.$ nFin $_{n}$ Pkts $\left.=0\right) \wedge\left(\right.$ nFinTcp $_{n}$ PktsTcp $=$ $0) \wedge(n P k t s=2) \wedge(n P k t s T c p=2) \wedge\left(\right.$ nRst $_{n}$ Pkts $=$ $0.5) \wedge\left(n\right.$ Syn $_{n}$ Pkts $\left.=0.5\right) \wedge\left(n\right.$ SynTcp $_{n}$ PktsTcp $\left.=0.5\right)$

Anomaly [44] appears in Figure 2 on subspaces (b) with the black cluster corresponding to $n S y n_{n} P k t s=1$ and (c), with the pink cluster corresponding to $n R_{s t}$ Pkts $=1$.

Finally, the algorithm generated the following signature that fully characterizes anomaly [44]:

\section{Characteristics of attack [44]:}

$($ bpp $=40) \wedge\left(\right.$ nBytes ${ }_{n}$ Flow $\left.=40\right) \wedge$ $(n$ DiffDestAddr $=1) \wedge($ PPkts $=1) \wedge\left(n\right.$ Pkts ${ }_{n}$ Flow $=$ $1) \wedge(n P k t s T c p=1) \wedge\left(\right.$ RRst $_{n}$ Pkts $\left.=1\right) \wedge\left(n\right.$ Syn $n_{n}$ Pkts $\left.=0\right)$

\section{CONCLUSION}

This paper presents an unsupervised algorithm for classifying illicit traffic. This algorithm has several advantages compared to previous work: (i) it works in a completely unsupervised manner, what makes it able to work on top of any monitoring system, and directly usable, without preliminary configuration or knowledge. (ii) It combines robust clustering techniques to avoid classical issues of clustering algorithms, e.g. sensitivity to initial configuration, the required a priori indication of teh number of clusters to be identified, or the sensitivity of results when using less pertinent features. (iii) It automatically builds simple and small signatures fully characterizing attacks; theses signature can then be used in a filtering security device. (iv) It is designed to run in real time by making possible to take advantage of the parallelism of our clustering approach.

This algorithm thus opens new perspectives for performing a risk analysis in the Internet - taking advantage of honeypot traffic - and automatically configuring related filtering rules on routers, switches, or firewalls.

\section{ACKNOWLEDGEMENTS}

The author sincerely thanks Michel Cukier and Bertrand Sobesto for providing the traffic traces gathered on the honeypots of the University of Maryland. The author thanks Johan Mazel and Pedro Casas who have been first involved in the research work on the sub-space clustering algorithm applied to the full Internet traffic. The author also thanks Richard Turc who started this work on the analysis of the honeypot traffic during his master internship at LAAS. This work is supported by the ONTIC project, funded by the European commission under grant FP7-ICT-2013-11/619633.

\section{REFERENCES}

[1] J. Mazel, P. Casas, Y. Labit, and P. Owezarski, "Sub-space clustering, interclustering results association \& anomaly correlation for unsupervised network anomaly detection," in 7th International Conference on Network and Service Management (CNSM 2011), CNSM'11, october 2011.

[2] R. Berthier, M. Cukier, M. Hiltunen, D. Kormann, G. Vesonder, and D. Sheleheda, "Nfsight: Netflow-based network awareness tool," in Proceedings of the 24th international conference on Large installation system administration (LISA'10), 2010.

[3] J. Mazel, "Unsupervised network anomaly detection," in PhD thesis of INSA Toulouse, 2011.
[4] P. Barford, J. Kline, D. Plonka, and A. Ron, "A signal analysis of network traffic anomalies," in Proc. ACM IMW, 2002.

[5] J. Brutlag, "Aberrant behavior detection in time series for network monitoring," in Proc. 14th Systems Administration Conference, 2000.

[6] A. Lakhina, M. Crovella, and C. Diot, "Characterization of networkwide anomalies in traffic flows," in Proc. ACM IMC, 2004.

[7] A. Lakhina, C. Diot, and M. Crovella, "Diagnosing network-wide traffic anomalies," in Proc. ACM SIGCOMM, 2004.

[8] A. Lakhina, M. Crovella, and C. Diot, "Mining anomalies using traffic feature distributions," in Proc. ACM SIGCOMM, 2005.

[9] B. Krishnamurthy, S. Sen, Y. Zhang, and Y. Chen, "Sketch-based change detection: Methods, evaluation, and applications," in Proc. ACM IMC, 2003.

[10] X. Li, F. Biang, M. Crovella, C. Diot, R. Govindan, G. Iannaccone, and A. Lakhina, "Detection and identification of network anomalies using sketch subspaces," in Proc. ACM IMC, 2006.

[11] H. Ringberg, A. Soule, J. Rexford, and C. Diot, "Sensitivity of pca for traffic anomaly detection," in Proc. ACM SIGMETRICS, 2007.

[12] P. Casas, S. Vaton, L. Fillatre, and I. Nikiforov, "Optimal volume anomaly detection and isolation in large-scale ip networks using coarsegrained measurements," in Computer Networks, vol. 54, pp. 1750-1766, 2010.

[13] P. Casas, J. Mazel, and P. Owezarski, "Unada: Unsupervised network anomaly detection using sub-space outliers ranking," in IFIP Networking conference, 2011.

[14] L. Portnoy, E. Eskin, and S. Stolfo, "Intrusion detection with unlabeled data using clustering," in Proc. ACM DMSA Workshop, 2001.

[15] E. Eskin, A. Arnold, M. Prerau, L. Portnoy, and S. Stolfo, "A geometric framework for unsupervised anomaly detection: Detecting intrusions in unlabeled data," in Applications of Data Mining in Computer Security, Kluwer Publisher, 2002.

[16] K. Leung and C. Leckie, "Unsupervised anomaly detection in network intrusion detection using clustering," in Proc. ACSC05, 2005.

[17] G. Fernandes and P. Owezarski, "Automated classification of network traffic anomalies," in Proc. SecureComm'09, 2009.

[18] F. Silveira and C. Diot, "Rca: Pulling anomalies by their root causes," in Proc. IEEE INFOCOM, 2010.

[19] G. Cormode and S. Muthukrishnan, "What's new: Finding significant differences in network data streams," in IEEE Trans. on Networking, vol. 13 (6), pp. 1219-1232, 2005.

[20] L. Parsons, E. Haque, and H. Liu, "Subspace clustering for high dimensional data: a review," in ACM SIGKDD Expl. Newsletter, vol. 6 (1), pp. 90-105, 2004

[21] A. Fred and A. K. Jain, "Combining multiple clusterings using evidence accumulation," in IEEE Trans. Pattern Analysis and Machine Intelligence, vol. 27 (6), pp. 835-850, 2005.

[22] A. K. Jain, "Data clustering: 50 years beyond k-means," in Pattern Recognition Letters, vol. 31 (8), pp. 651-666, 2010.

[23] A. Strehl and J. Ghosh, "Cluster ensembles - a knowledge reuse framework for combining multiple partitions," in Journal on Machine Learning Research, vol. 3, pp. 583-617, 2002.

[24] R. Agrawal, J. Gehrke, D. Gunopulos, and P. Raghavan, "Automatic subspace clustering of high dimensional data for data mining applications," in Proc. ACM SIGMOD, 1998. 